\title{
Classifying visual field loss in glaucoma through baseline matching of stable reference sequences
}

Meng, Shuanghui; Turpin, Andrew; Lazarescu, Mihai; Ivins, Jim

https://researchrepository.rmit.edu.au/esploro/outputs/9921861827201341/filesAndLinks?institution=61RMIT_INST\&index=null

Meng, S., Turpin, A., Lazarescu, M., \& Ivins, J. (2005). Classifying visual field loss in glaucoma through baseline matching of stable reference sequences. Proceedings of 2005 International Conference on Machine Learning and Cybernetics. https://doi.org/10.1109/ICMLC.2005.1527581

Published Version: https://doi.org/10.1109/ICMLC.2005.1527581

Repository homepage: https://researchrepository.rmit.edu.au

(c) 2005 IEEE. Personal use of this material is permitted. However, permission to reprint/republish this material for advertising or promotional purposes or for creating new collective works for resale or redistribution to servers or lists, or to reuse any copyrighted component of this work in other works must be obtained from the IEEE.

Downloaded On 2023/04/26 19:01:04 +1000 


\title{
CLASSIFYING VISUAL FIELD LOSS IN GLAUCOMA THROUGH BASELINE MATCHING OF STABLE REFERENCE SEQUENCES
}

\author{
SHUANG-HUI MENG ${ }^{1}$, ANDREW TURPIN ${ }^{2}$, MIHAI LAZARESCU ${ }^{1}$, JIM IVINS $^{1}$ \\ ${ }^{1}$ Department of Computing, Curtin University of Technology, GPO Box U1987, Perth 6845, Western Australia \\ ${ }^{2}$ School of Computing Science \& Information Technology, GPO Box 2476V, Melbourne, Victoria 3001, Australia \\ E-MAIL: \{sharonm, lazarescu, jim\}@cs.curtin.edu.au, aht@cs.rmit.edu.au
}

\begin{abstract}
:
Glaucoma is a common disease of the eye that often results in partial blindness. The main symptom of glaucoma is progressive loss of sight in the visual field over time. The clinical management of glaucoma involves monitoring the progress of the disease using a sequence of regular visual field tests. However, there is currently no universally accepted standard method for classifying changes in the visual field test data. Sequence matching techniques typically rely on similarity measures. However, visual field measurements are very noisy, particularly in people with glaucoma. It is therefore difficult to establish a reference data set including both stable and progressive visual fields. This paper proposes a method that uses a "baseline" computed from a query sequence, to match stable sequences in a database of visual field measurements collected from volunteers. The purpose of the new method is to classify a given query sequence as being stable or progressive. The results suggest that the new method gives a significant improvement in accuracy for identifying progressive sequenses, though there is a small penalty for stable sequences.
\end{abstract}

Keywords:

Sequence Matching; Visual Field; Glaucoma Change Probability; Confidence Interval

\section{Introduction}

Glaucoma is a common eye disease that affects the optic nerve. Its prevalence in Australia is about 3\% [1, 2]. Most people with glaucoma have no symptoms until some blindness occurs. A common feature in all patients is that the optic nerve fibers are damaged irreversibly [3]; however, with treatment the damage process can at least be slowed. Early detection of the disease is therefore crucial. If a patient continues to lose visual function after treatment, the glaucoma is said to be progressing, otherwise it is said to be stable.

Accurate evaluation of visual function is a crucial aspect of glaucoma management. It requires a series of visual field tests as described in the next section. However, it is difficult to separate true visual field loss from fluctuations that arise from learning effects, fatigue, and the inherent variation in the tests [4]. The high level of "noise" makes the correct diagnosis of glaucoma and the detection of progression difficult. A number of mathematical, statistical, and data mining methods have been proposed to determine visual field progression [5-10]. At present, however, there is no universally accepted standard against which to validate them [11]. Different clinical trials use different definitions of "progressing". Nevertheless, all agree that visual field measurement is an essential tool for detecting progression.

Sequence matching techniques have been widely used in applications such as image recognition, DNA analysis, signal processing, and anomaly detection for computer security [12, 13]. This study investigates sequence matching techniques applied to glaucomatous visual fields. This problem is complex because of the noise in the raw data, and the lack of a universally accepted standard for detecting progression. Hence we propose a method using baselines (Section 4) to match a database of stable sequences. The aims of this work are as follows: (1) for a given query sequence, to find the closest matches in a set of reference sequences and then classify the query sequence using the matches; (2) to compare the performance of the new method with the Glaucoma Change Probability (GCP) method that is widely used by clinicians to identify visual field loss.

The rest of this paper is organized as follows. Section 2 briefly describes the standard technique for measuring visual fields. The data sets used in this paper are described in Section 3. Section 4 describes the methods. The results are summarized in Section 5, and conclusions are presented in Section 6.

\section{Visual Field Measurement}

Regular eye tests are essential to detect glaucoma early 
and prevent significant loss of sight. The Standard Automated Periphery (SAP) technique currently employed in glaucoma management requires subjects to place their chins on an immobile stand and fixate on a central spot. Subjects are then asked to press a button whenever they see a flash of light. Lights of varying intensity are shown in each of 76 locations in the visual field. The marginally visible light intensity is recorded as the threshold sensitivity value. Each of the 76 locations has a threshold value reported in decibels $(\mathrm{dB})$, printed on a map of the visual field [14]. The threshold values range from 0 to $40 \mathrm{~dB} .0 \mathrm{~dB}$ indicates that the brightest light could not be seen - in other words, that the location is blind. Threshold values of 35-40 $\mathrm{dB}$ indicate exceptional vision at those locations. Figure 1 shows a visual field with localized loss in the top left corner. There are two physiological blind spots, as indicated by small zeros. For convenience in data processing, all visual fields of the left eye are reflected into the right eye format.

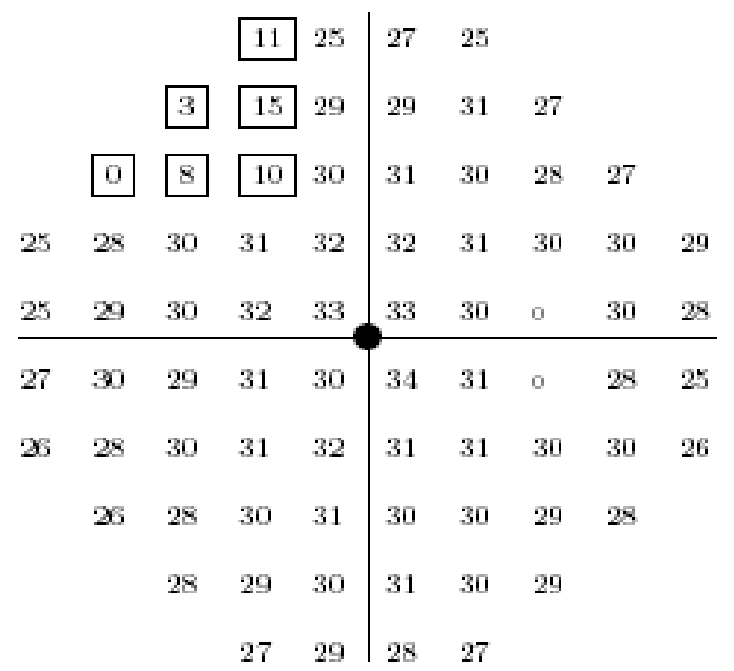

Figure 1. A map of the 76 locations in a SAP visual field showing localized loss in the top left quadrant.

When visual field measurements are repeated over time, the key task for an ophthalmologist is to determine whether change has occurred. This is a classification problem: each location must be classified as either nonprogressing (which includes stable and improving) or progressing. Several techniques exist to aid the clinician in this task. The most widely used of these is the Glaucoma Change Probability method described in Section 4.

\section{Data}

The proposed sequence matching method is based on a reference data set consisting only of stable sequences. To test the method, we created two artificial datasets to simulate glaucomatous progression and stable glaucoma. All of the data sets are described below.

\subsection{Reference Data Set}

The SAP data in the reference set were taken from 15 subjects with normal eyes and 35 subjects with stable glaucoma. The subjects ranged from 34 to 82 years of age (average, 60.9 years). The first five follow-up visual fields for each subject were used, even in cases where subjects had more than five SAP tests. Hence a total of 250 visual fields were analyzed. The time between tests for patients with stable glaucoma was typically about one week. The test interval for subjects with normal eyes was six months.

Together, these subjects provide $50 \times 74=3700$ sequences measured at locations which are known to be stable. For a stable sequence, because of noise in the raw date, the best overall estimate of the threshold value is the mean of the five measurements for that sequence. The distribution of the 3700 means is shown in Figure 2.

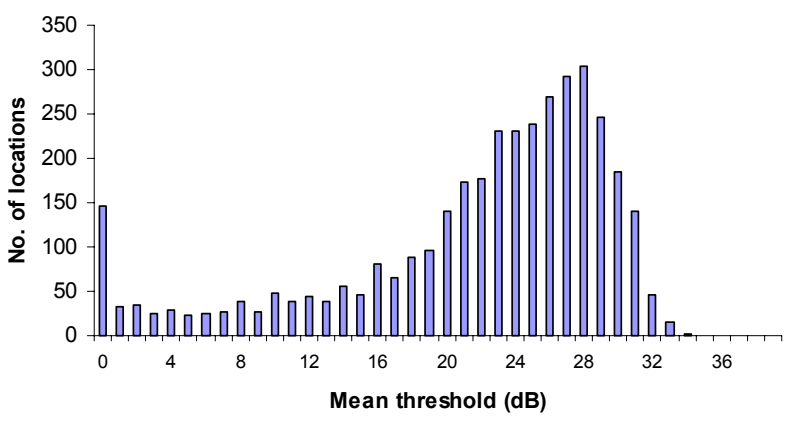

Figure 2. Distribution of the means of five visual field measurements at all 74 SAP locations, in 50 subjects.

Figure 2 shows that the data covered the entire range of stable visual field values $(0-34 \mathrm{~dB})$. All sequences were used to form a reference data set R. Let $R=\left\{S_{l}, S_{2}, \ldots, S_{n}\right\}$ denote the set of sequences where $n=3700$, and $S_{i}=\left\{y_{i l}\right.$, $\left.y_{i 2}, \ldots, y_{i 5}\right\}(i=1,2, \ldots, 3700)$ is a series of threshold values for one location of either a normal eye or a stable glaucomatous eye.

\subsection{Simulated Data Sets}

Spry et al $[15,16]$ describe an approach to generate stable and progressive sequences using a model based on variables: eccentricity-related fluctuation (location-based noise), and defect-related fluctuation (age-based noise). To test the effectiveness of the sequence matching method, we 
created a synthetic dataset using a simulation similar to that of Spry et al. This provided stable, linear, bilinear, and convex exponential degradation of locations over time. All simulated visual fields were in right eye format.

\subsubsection{Progressive Visual Field Data}

To simulate progressive visual field data, the first visual fields of 15 normal subjects were randomly distributed into three groups, each of five fields. All 15 visual fields were used as the initial values in the simulation. The visual field values in the three groups were then duplicated, but with $12 \mathrm{~dB}, 18 \mathrm{~dB}$, or $24 \mathrm{~dB}$ subtracted from all locations. These new fields were used as the final values. If the final value at any point was less than zero, this value was replaced by $0 \mathrm{~dB}$. The standard deviation of long-term fluctuation (with normal distribution) was $1 \mathrm{~dB}$. The standard deviation of short-term fluctuation was varied as $\left|x_{n}-N\right| \times 0.4 / 5$ [16]. Finally, five new visual fields were interpolated between the initial and the final fields, with average decreases of $2 \mathrm{~dB}, 3 \mathrm{~dB}$ or $4 \mathrm{~dB}$ between consecutive fields for the 3 groups respectively. The linear, bilinear and concave exponential procedures were applied to each of the 15 visual fields, providing a total of 45 progressive visual fields.

\subsubsection{Stable Visual Field Data}

To simulate stable visual field data, the first visual field from each of the 50 real subjects was used as both the first and last values. To generate the middle five visual fields, short-term and long-term fluctuations were used as described above. Thus, 50 virtual eyes with stable glaucoma were generated.

To avoid repetition, the initial and final values were removed from the simulated data. The number of sequences simulated in each group is shown in Table 1.

Table 1. The number of simulated sequences in each group

\begin{tabular}{|c|lcc|c|}
\hline \multicolumn{5}{|c|}{$(5 \times 74=370)}$. \\
\hline Progressing 12dB & Linear & Bilinear Convex & Total \\
Progressing 18dB & 370 & 370 & 370 & 1110 \\
Progressing 24dB & 370 & 370 & 370 & 1110 \\
Stable & 3700 & 0 & 370 & 1110 \\
\hline Sum & 4810 & 1110 & 1110 & 7030 \\
\hline
\end{tabular}

\section{Methods}

The 7030 simulated sequences (95 virtual patients) were evaluated using the GCP method with the established confidence intervals, and using the proposed matching method.

\subsection{GCP Methods}

Given a sequence of $n$ threshold measurements: $X=$ $\left\{x_{1}, x_{2}, \ldots, x_{n}\right\}$, the baseline is defined as $\left(x_{1}+x_{2}\right) / 2$ where $x_{1}$ and $x_{2}$ were taken in a short period so that no natural decline or glaucomatous progression occurred.

Glaucoma Change Probability (GCP) analysis calculates the difference between a threshold measurement and a baseline on a point-by-point basis, and then determines whether the difference falls inside or outside the 95\% confidence interval established from a database of stable glaucomatous visual fields [17]. If the difference for one location is less than the lower limit of the confidence interval, this location is said to be progressing. If the difference falls inside the confidence interval, this location is said to be stable. Otherwise, it is said to improving.

We present two methods of building a confidence interval by using the reference set $\mathrm{R}$. One method is similar to that used by Spry et al [11].

(1) The mean of all five values in a sequence: $\left\{y_{1}+y_{2}+\right.$ $\left.y_{3}+y_{4}+y_{5}\right\} / 5$ is calculated, and then rounded to the nearest integer. These integers are used to divide the sequences into groups.

(2) The difference between test and retest values is calculated for each sequence in each group. That is, $y_{i}-y_{i+l}$, $i=1,2,3,4$.

(3) The differences in each group are sorted from the smallest to the largest, and then the $2.5 \%$ and $97.5 \%$ percentiles are computed to form a $95 \%$ confidence interval for that group. This method for building a confidence interval is called SA in this paper.

Because there is no consensus as to how the underlying confidence interval should be derived, we modified the second step by taking $\left(y_{1}+y_{2}\right) / 2-y_{i},(i=3,4$, $5)$ instead of $y_{i}-y_{i+1}$. This method for building a confidence interval is called SB in this paper.

\subsection{Baseline Matching Stable Sequences}

Let query $Q=\left\{x_{1}, x_{2}, \ldots, x_{m}\right\}$ be a sequence of values for one location, where $x_{i}$ is the $i$ th value, and $m$ is the number of measurements. We are not able to find a sequence in $\mathrm{R}$ to match $\mathrm{Q}$ exactly due to noise, and because all reference sequences in $\mathrm{R}$ are stable, whereas the query sequence may be progressing. Thus, in this study we used a similarity measure $D$ :

$$
D=\mid \text { baseline }-\overline{S_{i}} \mid \leq \text { cutoff }
$$


Where $\overline{S_{i}}$ is the average of five visual field measurements from a stable sequence, and the baseline is from Q. This function is used to choose the best matching reference sequences in $\mathrm{R}$ for the query sequence $\mathrm{Q}$.

The rationale behind this method is that the baseline value for each query sequence $\mathrm{Q}$ is an observation of the initial condition, and is the comparison condition for future follow-up examinations [14]. For a stable sequence $\mathrm{S}_{i}=\left\{y_{i l}\right.$, $\left.y_{i 2}, \ldots, y_{i j}\right\}, \overline{S_{i}}$ is an unbiased estimate of the population mean at the corresponding location. Therefore, baseline $-\overline{S_{i}} \mid$ indicates that for a given query $\mathrm{Q}$, we select stable sequences which are the closest to the baseline of $\mathrm{Q}$. The degree of similarity depends on the cutoff.

For a given cutoff and query Q, we collected all points from matched sequences and sorted them from the smallest to the largest. The $2.5 \%$ and $97.5 \%$ percentiles in this range were calculated to form a $95 \%$ interval. If the $m$ th value $x_{m}$ in Q fell into the $95 \%$ interval, the query sequence was said to be stable. If $x_{m}$ was less than the lower limit of the interval, the query sequence was said to be progressing. Otherwise, the sequence was improving. Improving and stable sequences were classified together as nonprogressing.

\subsection{Experiments}

In the experiments, the first interpolated field was taken as the baseline for each query. That is, for a query sequence $Q=\left\{x_{1}, x_{2}, \ldots, x_{m}\right\}, x_{l}$ was the baseline value.

In this study, we only discuss baseline values from 16 to $31 \mathrm{~dB}$ due to the sample obtained from the stable visual fields. This is because of the small numbers below 16 (see Figure 2). The evaluation of an interval requires a sample size large enough. If the sample is too small, the method may not be reliable [18]. The formula for calculating the required sample size $n$ is

$$
n=Z^{2} \times \sigma^{2} / E^{2}
$$

where $E$ is the maximum allowable error (the difference between the population mean and the sample mean). $Z$ is obtained by using the given confidence interval coefficient $\alpha$, and $\sigma$ is the population standard deviation. For this study, $E=1, Z=1.96$ (computed by using $\alpha=0.95$ ), and $\sigma$ is computed using Henson's [19] standard deviation:

$$
\log _{e}(S D)=-0.081 d B+3.27
$$

In equation (1), we used values between 0.1 and 1.0 as the cutoff to select matches in $\mathrm{R}$. The results are examined in Section 5.

\subsection{Statistical Methods}

Both the new method SB and the GCP method SA were applied to the simulated visual fields. For each simulated sequence, each method produced a binary outcome: classified correctly or not. Cochran's Q-test [20] was used to asses whether any difference between the methods was due to chance. This test is basically an ANOVA for data that is binary (also referred to as dichotomous or categorical). If the Q-test indicated a difference between the methods, then a non-parametric pairwise comparison technique outlined in Sheskin [21, page 689] was applied. This takes into account a correction for multiple $(\mathrm{k} \geq 2)$ comparisons.

\section{Results}

Figures 3 and 4 show the results obtained by the new method on the simulated datasets for classifying the $3 r d$, 4th and 5th visual field measurements, baseline values being 16 to $31 \mathrm{~dB}$, at different cutoffs. When the cutoff gradually increases, the accuracy of classification gradually increases for stable sequences, while it gradually decreases for progressing sequences. This is because when the cutoff is less than 0.4 , some query sequences cannot get enough points as required by equation (2). For these sequences, the result of classification is not reliable. On the other hand, when the cutoff is greater than 0.6 , query sequences may obtain enough points from the matched stable sequences. However, the means of some matched stable sequences are closer to the integer "baseline +1 " or "baseline -1 ". (| baseline - mean $\mid \leq$ cutoff $\Rightarrow$ baseline - cutoff $\leq$ mean $\leq$ baseline + cutoff). That is, the interval formed by taking all points from matched stable sequences is too wide. When the cutoff is 0.4 , the minimum number of points from the matched sequences is 120 to satisfy equation (2). Figure 3 and 4 show that when the cutoff is $0.4,0.5$, or 0.6 , the results of correct classification are very similar for classifying the $3 r d$, 4 th or 5 th visual field.

We took the cutoff as 0.5 to compare the GCP methods. As a comparison for each location, we also used the GCP criteria: (1) for a given location, if progression at this location was identified in two of three consecutive fields (denoted by 2of3), this location was said to be progressing; or (2) in three of three consecutive fields (denoted by 3of3) [22]. The results are shown in Table 2 using 5 visual field measurements. 


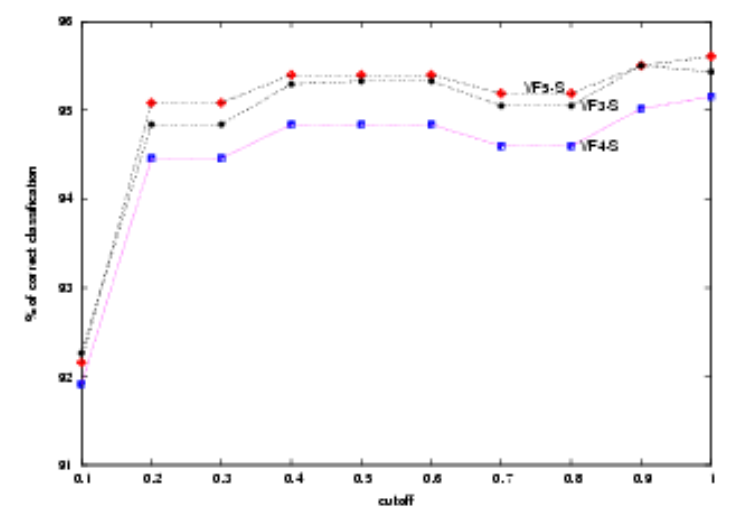

Figure 3. The percentage of correct classification for stable sequences with baseline values from 16 to $31 \mathrm{~dB}$ at different cutoffs for the $3 r d$, $4 t h$ and $5 t h$ visual field measurements.

("S" for stable sequences.)

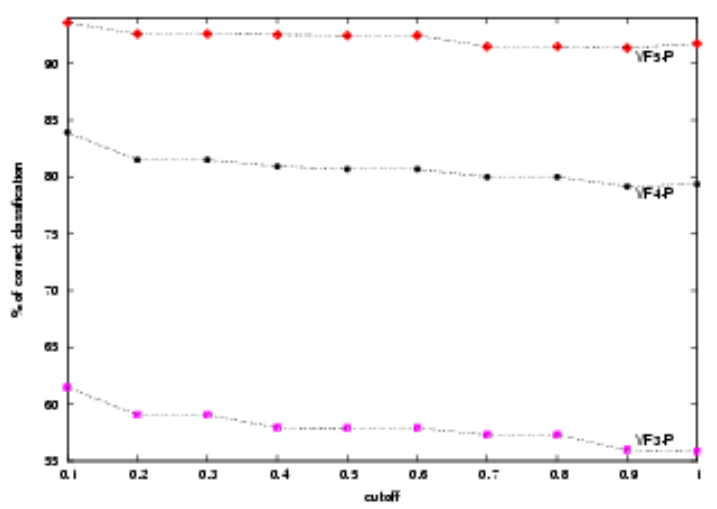

Figure 4. The percentage of correct classification for progressing sequences with baseline values from 16 to $31 \mathrm{~dB}$ at different cutoffs for the $3 r d$, $4 t h$ and $5 t h$ visual field measurements. ("P" for progressing sequences.)

In Table 2, "P" indicates the percentage of correct classification for progressing sequences, " $\mathrm{S}$ " for stable sequences. The new method offers higher accuracy for classifying progressing sequences, while accuracy is lower for stable sequences. Using Cochran's Q-test confidence level $\alpha$ of 0.05 , we analyzed whether the new method improves classification overall. A significant difference was found. Hence, pairwise comparisons were performed and are given in Table 3.

Table 2 and 3 show that the GCP method using confidence interval SB is statistically significantly more accurate compared with GCP using SA when classifying progressive sequences. The accuracy of GCP with SB in classifying stable sequences decreases slightly, but the loss is not statistically significant. The new method $\mathrm{MH}$ is the best for correctly classifying progressing sequences. It increases accuracy by between $8 \%$ and $14 \%$ compared with GCP using confidence interval SB and SA respectively. It decreases accuracy by less than $2 \%$ for detecting stable sequences when GCP criterion (1) was used. When GCP criterion (2) was used, the method offers about $11 \%$ and $16 \%$ increase respectively for progressing sequences, with about $0.5 \%$ decrease for stable sequences.

Table 2. The percentage of correct classification for baseline 16 to $31 \mathrm{~dB}$. SA and SB are confidence interval methods described in Section 4.1. MH denotes the new method proposed in Section 4.2.

\begin{tabular}{|c|cc|cc|cc|}
\hline \multirow{2}{*}{ Criterion } & SA & & SB & & MH & \\
\cline { 2 - 7 } & P & S & P & S & P & S \\
\hline 2of3 & 68.35 & 99.06 & 74.10 & 98.95 & 82.72 & 97.11 \\
3of3 & 36.24 & 99.83 & 41.52 & 99.79 & 52.99 & 99.30 \\
\hline
\end{tabular}

Table 3. Difference (in percent) of correct classification between methods. Statistically significant change is shown underlined $(\alpha<0.05)$.

\begin{tabular}{|c|l|lll|}
\hline Criterion & Group & $S B-S A$ & $M H-S A$ & $M H-S B$ \\
\hline \multirow{5}{*}{ 2of3 } & $12 \mathrm{~dB}$ & $\underline{+7.8}$ & $\underline{+21.7}$ & $\underline{+13.9}$ \\
& $18 \mathrm{~dB}$ & $\underline{+4.9}$ & $\underline{+12.1}$ & $\frac{+7.2}{+3.6}$ \\
& $24 \mathrm{~dB}$ & $\underline{+3.8}$ & $\underline{+7.4}$ & $\underline{+3.6}$ \\
& Stable & -0.1 & $\underline{-1.5}$ & $\underline{-1.4}$ \\
\hline \multirow{3}{*}{$30 f 3$} & $12 \mathrm{~dB}$ & $\underline{+3.8}$ & $\underline{+45.4}$ & $\underline{+41.6}$ \\
& $18 \mathrm{~dB}$ & $\underline{+5.9}$ & $\underline{+48.4}$ & $\underline{+42.6}$ \\
& $24 \mathrm{~dB}$ & $\underline{+5.4}$ & $\underline{+39.3}$ & $\underline{+33.9}$ \\
& Stable & 0.00 & $\underline{-2.1}$ & $\underline{-2.1}$ \\
\hline
\end{tabular}

Table 2 and 3 show that the GCP method using confidence interval SB is statistically significantly more accurate compared with GCP using SA when classifying progressive sequences. The accuracy of GCP with SB in classifying stable sequences decreases slightly, but the loss is not statistically significant. The proposed new method is the best for correctly classifying progressing sequences. It increases accuracy by between $8 \%$ and $14 \%$ compared with GCP using confidence interval SB and SA respectively. It decreases accuracy by less than $2 \%$ for detecting stable sequences when GCP criterion (1) was used. When GCP criterion (2) was used, the proposed method offers about $11 \%$ and $16 \%$ increase respectively for progressing sequences, with about $0.5 \%$ decrease for stable sequences.

\section{Conclusion}

This paper has described an application of sequence 
matching to the problem of classifying change in visual fields. It is difficult to establish a set of reference sequences which includes progressing sequences, because using different techniques can give different results for a given patient with progressing glaucoma. Therefore, we have focused on the use of the baseline for a given query sequence. In this way, the set of reference sequences can be constructed only from stable sequences. The results indicate that using the new method can significantly improve the accuracy of identifying progressing sequences, though there is a small penalty for stable sequences.

\section{Acknowledgements}

This research was supported by the Australian Research Council. The stable visual field data set used for constructing the reference sequences was provided by Prof. Bal Chauhan at Dalhousie University, Canada.

\section{References}

[1] Mitchell P, Smith W, Attebo K, Healey PR. Prevalence of open-angle glaucoma in Australia. The Blue Mountains Eye Study. Ophthalmology. 103: 1661-1669. 1996.

[2] Rochtchina E., Mitchell P. Projected number of Australians with glaucoma in 2000 and 2030. Clin Experiment Ophthalmol. 28:146-148. 2000.

[3] Johnson C. A., Sample P. A., Zangwill L. M., et al. Structure and function evaluation (SAFE): 2. comparison of optic disk and visual field characteristics. American Journal of Ophthalmology. 135:148-154. 2003.

[4] Flammer J., Drance S. M., Zulauf M., Differential light threshold. Arch Ophthalmol. 102: 704-706. 1984.

[5] Heijl A., Lindgren G., Olsson J., Asman P. Visual field interpretation with empiric probability maps. Arch Ophthalmol. 107:204-208. 1989.

[6] Katz J., Congdon N., Friedman D. S.. Methodological variations in estimating apparent progressive visual field loss in clinical trials of glaucoma treatment. Arch Ophthalmol. 117:1137-1142. 1999.

[7] Lazarescu M., Turpin A. Classifying glaucomatous progression using decision trees. Proceedings of IASTED International Symposia on Applied Informatics, Innsbruck, Austria. 205-210, 2003.

[8] Lin A., Hoffman D., Gaasterland D. E., Caprioli J. Neural networks to identify glaucomatous visual field progression. American Journal of Ophthalmology. 135:49-54. 2003.
[9] Morgan R. K., Feuer W. J., Anderson D. R. Statpac 2 glaucoma change probability Arch Ophthalmol. 109: 1690-1692. 1991.

[10] Turpin A., Frank E., Hall M., Witten I. H., Johnson C. A. Detecting progression in glaucoma using data mining techniques. Proceedings of the 5th Pacific Asia Conference on Knowledge Discovery and Data Mining, 136-147 2001.

[11] Spry P. G., Johnson C. A., Chauhan B. C. Identification of progressive glaucomatous visual field loss. Survey of Ophthalmology. 47: 158-173. 2002.

[12] Lane T., Brodley C. E. Sequence matching and learning in anomaly detection for computer security. AI Approaches to Fraud Detection and Risk Management (Fawcett, Haimowitz, Provost, Stolfo. eds.): 43-49 AAAI Press 1997.

[13] Navarro G. A guided tour to approximate string matching. ACM Computing Surveys. 33:31-38. 2001.

[14] Anderson D. R., Patella. V. M. Automated static perimetry. Mosby, second ed., ISBN:0815143842. 1999.

[15] Spry P. G., Bates A. B., Johnson C. A., Chauhan B. C. Simulation of longitudinal threshold visual field data. Investigative Ophthalmology and Visual Science. 41:2192-2200. 2000.

[16] Vesti E., Spry P. G., Chauhan B. C., Johnson C. A. Sensitivity differences between real patient and computer stimulated visual fields. Journal of Glaucoma. 11:35-45. 2002.

[17] Heijl A., Lindgren A., Lindgren G.. Test-retest variability in glaucomatous visual fields. American Journal of Ophthalmology. 108:130-135. 1989.

[18] Hughes A., Grawoig D. Statistics: A foundation for analysis. Addison-Wesley. ISBN: 76-133891. 1971.

[19] Henson D. B., Chaudry S., Artes P. H., Faragher E. B., Ansons A. Response variability in the visual field: comparison of optic neuritis, glaucoma, ocular hypertension, and normal eyes. Investigative Ophthalmology and Visual Science. 41:417-421. 2000.

[20] Cochran W. G. The comparison of percentages in matched samples. Biometrika. 37:256-266. 1950.

[21] Sheskin D. J. Handbook of parametric and nonparametric statistical procedures. Chapman \& Hall/CRC, second ed. 2000.

[22] Vesti E, Johnson C. A., Chauhan B. C. Comparison of different methods for detecting glaucomatous visual field progression. Investigative Ophthalmology and Visual Science. 44:3873-3879. 2003. 